\title{
Role of Transesophageal Echocardiography Diagnosis of an Acute Aortic Paravalvular Leak and Successful Treatment with Percutaneous Closure
}

\author{
Lingcan Tan ${ }^{1}$, Qiao $\mathrm{Li}^{2}$, Yuan Feng ${ }^{3}$, and Haibo Song ${ }^{4}$ \\ ${ }^{1}$ Affiliation not available \\ ${ }^{2}$ Sichuan University West China Hospital \\ ${ }^{3}$ West China Hospital, Sichuan University \\ ${ }^{4}$ Department of Anesthesiology
}

July 6, 2020

\begin{abstract}
Paravalvular Leaks are well-recognized complications after artificial valve replacement and sometimes can be fatal. Symptomatic periprosthetic leaks are closely associated with increased mobility and mortality. Herein, we report the use of transesophageal echocardiogram diagnosis of aortic paravalvular leak after aortic valve replacement and successful percutaneous closure of leak. This case report highlights the role of transesophageal echocardiography in diagnosis of aortic prosthesis leak, and confirms the validity of interventional transcatheter repair.
\end{abstract}

\section{Introduction}

As the number of surgical and transcatheter valve replacements continues to increase in the aging population, so does the prevalence of paravalvular leak (PVL), the uncommon but life-threatening complication. Although Given known guidelines focus on valvular heart disease and PVL, diagnosis of PVL is arduous and prone to misdiagnosis. Transesophageal echocardiogram (TEE) plays a major role in assessment of the location and severity of PVL. We report a case of an elderly man diagnosed as PVL with help of TEE, and ultimately received interventional transcatheter closure. Signed written informed consent from the patient was obtained for reporting this case.

\section{Case presentation}

A 72-year-old man was referred to our emergency room due to exertional dyspnea for 3 years, aggravation for 1 week. He had 3 years history of valvular heart disease, and herpes zoster of right leg 2 month back, home medication included bisoprolol, Atorvastatin and acyclovir. Abnormal elevated cardiac enzymes, including troponin level trending up to $52.5(0-14 \mathrm{ng} / \mathrm{L})$ and brain natriuretic peptide up to $4321(8-21 \mathrm{mg} / \mathrm{dL})$, suggested presence of congestive heart failure. Transthoracic echocardiography (TTE) had proven severe aortic valve regurgitation and mild aortic valve stenosis, with significantly dilated left ventricular of $81 \mathrm{~mm}$, and impaired left ventricular eject fraction of $39 \%$. Then the patient was admitted to cardiovascular surgery unit. On admission, initial vital signs showed a body temperature of 36.5 , blood pressure of $111 / 75 \mathrm{~mm}$ $\mathrm{Hg}$, respiration rate of 20 breaths/min, and heart rate of 68 beats per min. The patient received aortic valve replacement under cardiopulmonary bypass one week after admission, intraoperative TEE showed no paravalvular leak, postoperatively, was routinely transferred into intensive care unit.

During ICU, the patient showed continuous low cardiac output syndrome, even with large dose of cardiotonic. Postoperative TTE revealed dilated left ventricular of $73 \mathrm{~mm}$, and significantly reduced eject fraction of $30 \%$. 
Whereas, in the apical 5-chamber view, TTE detected dubious coronary flow with velocity of $2.8 \mathrm{~m} / \mathrm{s}$ in the left ventricular outflow tract (LVOT), which suspected coronary-left ventricular fistula, which neither monitored in preoperative TTE nor intraoperative TEE. Therefore, we performed a second TEE to him 3 days post-surgery, noticing a jet regurgitation in long axis view of aortic valve, which started outside prosthetic frame into LVOT, with high velocity of $4.41 \mathrm{~m} / \mathrm{s}$ and vena contract width of $4.7 \mathrm{~mm}$ (Figure 1a-1b). Short axis view of aortic valve also revealed the regurgitation was paravalvular, outside the edge of sewing ring (Figure 1c-1d). PVL was highly suspectable, then subsequent ascending aortic angiography confirmed it (Figure 2). PVL diameter was calculated by angiography, showing approximate $3 \mathrm{~mm}$ closed to aortic root. Afterwards, leak was passed by a hydrophilic terumo 0.32 inch guide wire and $5 \mathrm{~F}$ delivery catheter was placed into left ventricle, transcatheter occlusion was successfully made to intervene by preforming a $10 \mathrm{~mm}$ Vascular Plug device (Starway Medical Technology Inc., Cardi-O-Fix Plug Occluder )(Figure 3a). After device was in place, second aortic root angiography revealed no sign of PVL during opening of aortic side, neither of coronary obstruction (Figure 3b). 8 days after closure, TTE showed reduced left ventricular of $50 \mathrm{~mm}$, left ventricular motion synchrony, slightly increased EF of $38 \%$, as well as no observation of PVL. The patient had been discharged from hospital 26 day post-surgery, no signs of recurrence have been detected in 2 months of follow-up.

\section{Discussion}

Paravalvular leak has been demonstrated $2-10 \%$ in aortic prosthetic implantation and $7-17 \%$ in mitral position, respectively ${ }^{\{1\}}$. While majority of PVLs is subclinical, approximate 1-5\% PVL patients can develop serious clinical consequences, such as severe heart failure, as described in our case ${ }^{\{2\}}$. Though given available experiences and guidelines to assess and manage PVL, diagnosis and differential diagnosis of PVL may not be effortless like we imaged.

In our case, patient was once mistaken as coronary artery-left ventricular fistula, probably owing to limitation of acoustic shadowing, which could preclude visualization of aortic prosthetic components, resulting in misidentification of abnormal color doppler signal, and underestimate severity of regurgitation. Coronary artery fistula is an anomalous connection between coronary artery and cardiac chamber or great vessel, accounts for $0.2 \%-0.4 \%$ of congenital heart disease ${ }^{\{3\}}$. Majority of coronary fistula opens to the right heart, very rare in the left ventricular. 2D echocardiography shows dilated and tortuous coronary artery, and associated enlarged feeding artery, sometimes appearing the origin or course of the fistula vessel ${ }^{\{4-6\}}$. Color Doppler helps in detection of the entrance and termination site of drainage, characterized by continuous shunt both in systolic and diastolic period ${ }^{\{4\}}$. PVL, unlike, usually appears as abnormal jet flow outside prosthetic valve frame, no course of drainage. TEE can display heart structure more clearly than TTE in real time, help quantitatively evaluate heart function and heart structure, and be simpler, cheaper and less time-consuming than cardiac angiography. In addition, multi-modality imaging, including combination of TTE, TEE and cardiac angiography, may be needed to confirm and assess coronary fistula considering the complexity of diagnosis ${ }^{\{1,2\}}$. As in this case, TEE descriptions promote suspicion of PVL, and cardiac angiography confirmed it, stressing the utilization of multi-modality in PVL diagnosis.

Previously, clinically significant PVLs could be only corrected by reoperation, whereas, along with increased mortality closely related to reoperation. In recent two decades, transcatheter closure has evolved as available option for PVL patients, especially for those who are at high surgical risk and have suitable anatomic features for transcatheter closure ${ }^{\{1\}}$. In the literature, a large amount of PVL cases have been successfully closed with various occlude devices, despite that no device has been approved for PVL occlusion by FDA $\{1,2,7,8\}$. Available devices include the Amplatzer family of occluders/plugs (St Jude Medical, St Paul, MN, USA), Amplatzer septal occluder (ASO), Amplatzer muscular VSD occluder (mVSD), Amplatzer duct occluder (ADO), and Amplatzer vascular plugs (AVP II and III). On the other hand, successful closure require experienced interventional cardiologists to be familiar to complex catheter techniques and have facility to perform. Our present report is a successful case of repair of aortic paravalvular leakage with one kind of domestic Plug device.

\section{Conclusion}


Acute paravalvular aortic leak following aortic valve replacement is uncommon yet life-threatening complication. In this case, the patient showed significant manifestation of heart failure owing to PVL. TEE plays a major role in diagnosis of PVL and multi-modality imaging should be advocated. Interventional transcatheter closure had been shown as an appropriate option for repair of PVL.

\section{Footnote}

Conflicts of Interest: The authors have no conflicts of interest to declare.

\section{Reference}

1. Kliger Chad, Eiros Rocio, Isasti Guillermo et al. Review of surgical prosthetic paravalvular leaks: diagnosis and catheter-based closure. Eur. Heart J., 2013, 34: 638-49.

2. Bernard Samuel, Yucel Evin, Paravalvular Leaks-From Diagnosis to Management. Curr Treat Options Cardiovasc Med, 2019, 21: 67.

3. Said SA, Lam J, van der Werf T. Solitary coronary artery fistulas: A congenital anomaly in children and adults. A contemporary review. Congenit Heart Dis 2006;1:63-76.

4. Kumar Bhupesh, Kumar Alok, Kumar Ganesh et al. Role of transesophageal echocardiography in surgical retrieval of embolized amplatzer device and closure of coronary-cameral fistula. Ann Card Anaesth, 2017, 20: 351-354.

5. Chung P C, Huang P C, Liu Y F, et al. Transesophageal echocardiography, more than a diagnostic tool: use during surgical ligation of coronary artery fistulae - a case report. Journal of Cardiothoracic Surgery, 2012, 7(1):28.

6. Bittencourt MS, Seltman M, Achenbach S, Rost C, Ropers D. Right coronary artery fistula to the coronary sinus and right atrium associated with giant right coronary enlargement detected by transthoracic echocardiography. Eur J Echocardiogr 2011;12:E22-E22.

7. Quader N, Davidson C J, Rigolin V H . Percutaneous Closure of Perivalvular Mitral Regurgitation: How Should the Interventionalists and the Echocardiographers Communicate? Journal of the American Society of Echocardiography, 2015, 28(5):497-508.

8. Osken A, Aydin E, Akdemir R, et al. Percutaneous Closure of an Aortic Prosthetic Paravalvular Leak with Device in a Patient Presenting with Heart Failure. Heart Views, 2015, 16(2):56-58.

\section{Figure Legends}

Figure 1. TEE revealing prosthetic aortic valve regurgitation outside the edge of sewing ring both from long axis and short axis view. LA, left atrium; LV, left ventricle; RA, right atrium; RV, right ventricle; LVOT, left ventricular outflow tract; RVOT, right ventricular outflow tract; Ao, aorta; TEE, transesophageal echocardiogram.

Figure 2. Aortic root angiography confirming PVL. PVL, paravalvular leak.

Figure 3. Releasing of Vascular Plug device after positioning PVL (a) and aortic root angiography exclude PVL following closure (b). PVL, paravalvular leak. 

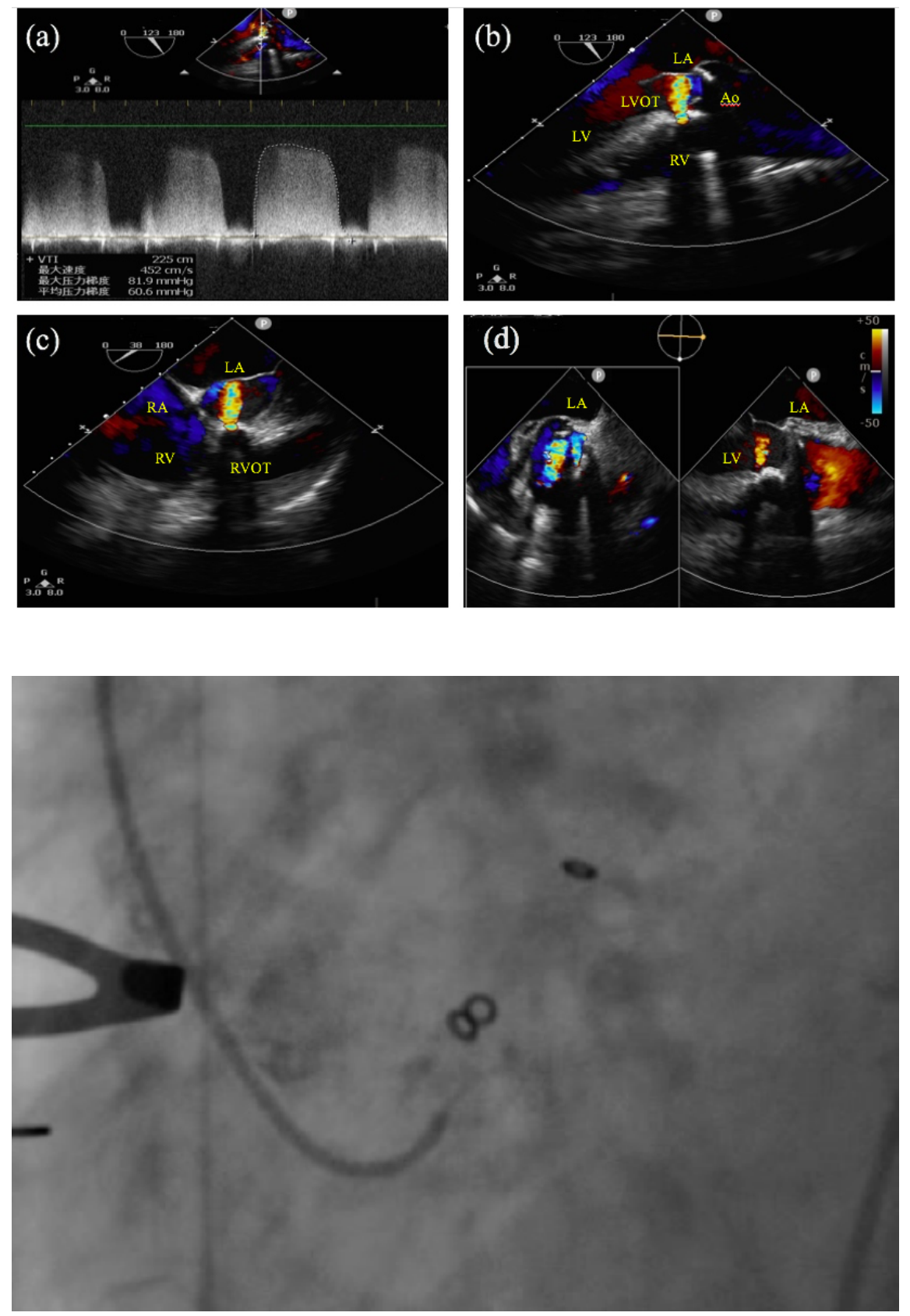


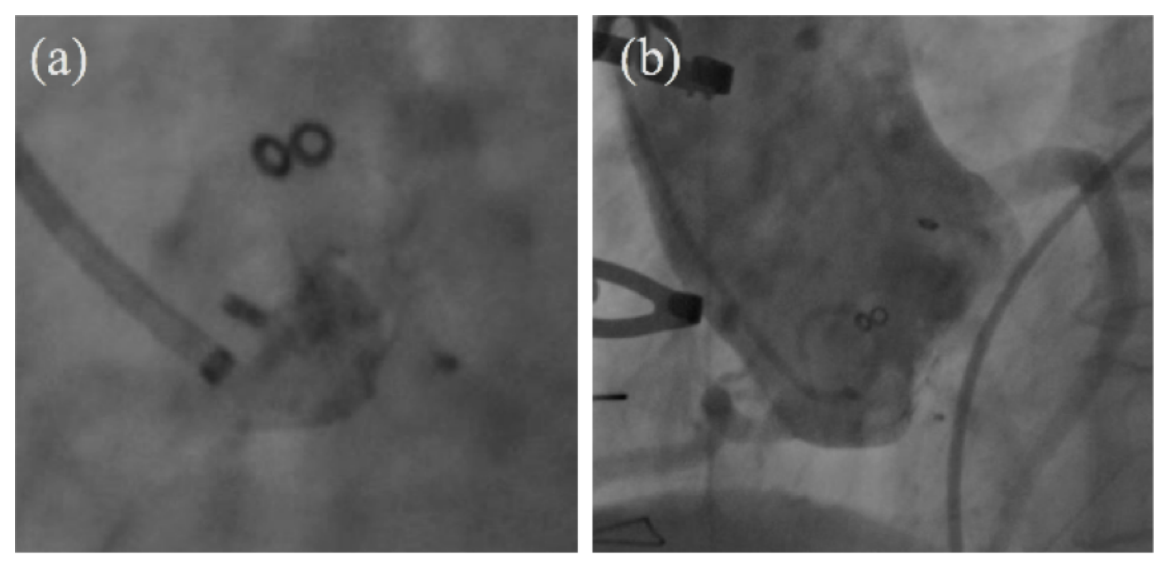

